\title{
Yttrium Y 90 Resin Microspheres
}

National Cancer Institute

\section{Source}

National Cancer Institute. Yttrium Y 90 Resin Microspheres. NCI Thesaurus. Code C64539.

An injectable formulation of the radioisotope yttrium Y 90 encapsulated in resin microspheres with potential antineoplastic activity. When injected into arterial vasculature supplying the tumor, yttrium $Y 90$ resin microspheres occlude tumor blood vessels and selectively deliver a cytotoxic dose of beta emitting yttrium Y 90 to the tumor site, which may result in tumor cell death and tumor regression. 